\title{
Resource Review \\ World Wide Web Resources on Obstetrical and Gynecological Infections
}

\author{
Stavros Athanasiou, ${ }^{1,2}$ Eleni Pitsouni, ${ }^{1}$ Christos lavazzo, ${ }^{1}$ \\ Eirinaios M. Karamanis, ${ }^{1}$ and Matthew E. Falagas ${ }^{1,3}$ \\ ${ }^{1}$ Alfa Institute of Biomedical Sciences, 9 Neapoleos Street, 15123 Marousi, Athens, Greece \\ ${ }^{2} 1$ st Department of Obstetrics and Gynecology, Athens University School of Medicine, Athens, Greece \\ ${ }^{3}$ Department of Medicine, Tufts University School of Medicine, Boston, MA 20110, USA
}

Correspondence should be addressed to Matthew E. Falagas, m.falagas@aibs.gr

Received 29 June 2007; Accepted 9 July 2007

\begin{abstract}
Modern information and communications technology has provided medical students and practitioners around the world with a new, valuable, and easy-to-use way to retrieve potentially useful information. Using previously described by our research group methodology, we generated a list of 50 Internet resources in the field of obstetrical and gynecological infections. We believe that the availability of such a list will help in the education of students and clinicians interested in obstetrical and gynecological infections.
\end{abstract}

Copyright (C) 2007 Stavros Athanasiou et al. This is an open access article distributed under the Creative Commons Attribution License, which permits unrestricted use, distribution, and reproduction in any medium, provided the original work is properly cited.

Nowadays the need for obtaining updated medical information is essential to doctors in practice. The fast progress in medical science is a fact that cannot be ignored. This statement also applies to the field of infectious diseases, including obstetrical and gynecological infections. For example, the recent introduction of the vaccine for human papilloma virus (HPV) represents a major advance in the battle against cervical cancer. The interest on obstetrical infections is of paramount importance because of the effects such infections have on pregnant women as well as on fetuses and newborns. Therefore, consultation of appropriate educational resources on diagnostic tests, treatment, and management options, in general, on obstetrical and gynecological infections is useful to practitioners, especially obstetricians and gynecologists.

Under these circumstances, the World Wide Web may be seen as a very useful educational tool for practitioners and students. It provides quick and easy access to information of different kinds, including medical knowledge. In many occasions, information provided by World Wide Web sources is updated frequently (monthly, weekly, or even daily). Although, there are, many popular and well-known sites that can provide useful medical information, a significant number of them are not widely in use.

The purpose of this contribution is to compile a list of World Wide Web sites that include information regarding obstetrical and gynecological infections see Table 1. All of the sites that we included in our list are free-accessed and in English. We did not include in our list of World Wide Web sites well-known worldwide valuable resources such as PubMed. The methodology we used in this paper has been described in previous relevant contributions of our team [1-3]. An effort was made to gather as many sites as possible referring to obstetrical and gynecological infections. However, we recognize that some sites that offer valuable information on this issue were overlooked. We think that the list of World Wide Web sites may be found useful by practicing obstetricians and gynecologists around the world, especially those clinicians with Internet access practicing in developing countries.

TABLE 1: World Wide Web resources on obstetrical and gynecological infections.

\begin{tabular}{lll}
\hline Website title & Website address & Comments \\
\hline WHO & http://www.who.int/topics/en & $\begin{array}{l}\text { Links and guidelines for surveillance, pre- } \\
\text { vention, of management and a treatment } \\
\text { of ob/gyn infections }\end{array}$ \\
\hline
\end{tabular}


TABle 1: Continued.

\begin{tabular}{|c|c|c|}
\hline Website title & Website address & Comments \\
\hline $\begin{array}{l}\text { Centers for disease control and } \\
\text { prevention }\end{array}$ & http://www.cdc.gov/std & $\begin{array}{l}\text { Information about prevention, surveil- } \\
\text { lance, and treatment of STD }\end{array}$ \\
\hline $\begin{array}{l}\text { European center for disease } \\
\text { prevention and control }\end{array}$ & http://www.eurosurveillance.org/index-02.asp & $\begin{array}{l}\text { Surveillance of communicable infections } \\
\text { across Europe }\end{array}$ \\
\hline $\begin{array}{l}\text { The American College of Ob- } \\
\text { stetrics and Gynecologists }\end{array}$ & http://www.acog.org & $\begin{array}{l}\text { Information about infections and many } \\
\text { useful links }\end{array}$ \\
\hline $\begin{array}{l}\text { Royal College of Obstetricians } \\
\text { and Gynaecologists }\end{array}$ & http://www.rcog.org.uk & Clinical guidance on ob/gyn infections \\
\hline $\begin{array}{l}\text { The Society of Obstetrics and } \\
\text { Gynecologists of Canada }\end{array}$ & http://www.sogc.org/guidelines/index_e.asp & Clinical practice guidelines \\
\hline Obgyn.net & http://www.obgyn.net/search/search.asp & $\begin{array}{l}\text { Information about infections and useful } \\
\text { links }\end{array}$ \\
\hline Lincoln center & http://www.lincolncenterobgyn.com/links.htm & Links to ob/gyn sites \\
\hline $\begin{array}{l}\text { Infectious diseases in obstet- } \\
\text { rics and gynaecology }\end{array}$ & http://www.hindawi.com/journals/idog & $\begin{array}{l}\text { An open access journal with special inter- } \\
\text { est in ob/gyn infections (free access) }\end{array}$ \\
\hline $\begin{array}{l}\text { The internet journal of gynae- } \\
\text { cology and obstetrics }\end{array}$ & $\begin{array}{l}\text { http://www.ispub.com/ostia/index.php?xmlFilePath } \\
=\text { journals/ijgo/front.xml }\end{array}$ & Online free-access journal (free access) \\
\hline Contemporary ob/gyn & http://www.contemporaryobgyn.net/obgyn & Full articles about ob/gyn infections \\
\hline $\begin{array}{l}\text { British Columbia Ministry of } \\
\text { Health }\end{array}$ & http://www.bchealthguide.org/womens.stm & $\begin{array}{l}\text { Online guide of women's health including } \\
\text { useful links }\end{array}$ \\
\hline $\begin{array}{l}\text { Pan American Health Organ- } \\
\text { ization }\end{array}$ & http://www.paho.org & $\begin{array}{l}\text { Links to sites about the prevention and } \\
\text { management of communicable diseases } \\
\text { and their surveillance }\end{array}$ \\
\hline National Library for Health & http://www.library.nhs.uk/?autoLogin=0 & $\begin{array}{l}\text { Evidence-based reviews, guidance, and } \\
\text { specialist libraries }\end{array}$ \\
\hline $\begin{array}{l}\text { University of California, De- } \\
\text { partment of Medicine }\end{array}$ & http://medicine.ucsf.edu/resources/guidelines & $\begin{array}{l}\text { Links to sites that provide clinical } \\
\text { guidelines }\end{array}$ \\
\hline $\begin{array}{l}\text { The University of Iowa Li- } \\
\text { braries }\end{array}$ & http://www.lib.uiowa.edu/hardin/md/obgyn.html & $\begin{array}{l}\text { Links to images, symptoms, and treat- } \\
\text { ment for ob/gyn infections }\end{array}$ \\
\hline $\begin{array}{l}\text { North Carolina, School of } \\
\text { Medicine }\end{array}$ & http://www.med.unc.edu/obgyn & $\begin{array}{l}\text { Updated information for obstetricians } \\
\text { and gynaecologists }\end{array}$ \\
\hline $\begin{array}{l}\text { Kirksville College of Oste- } \\
\text { opathic Medicine, Faculty of } \\
\text { Microbiology andImmunolo- } \\
\text { gy }\end{array}$ & http://www.kcom.edu/faculty/chamberlain/std.htm & $\begin{array}{l}\text { Symptoms, diagnosis, and treatment of } \\
\text { STD }\end{array}$ \\
\hline University of Alberta & $\begin{array}{l}\text { http://www.obgyn.med.ualberta.ca/pdf/0405Talks/ } \\
\text { Turnell/Peri_Infs_2004.pdf }\end{array}$ & Lecture slides about perinatal infections \\
\hline $\begin{array}{l}\text { University of Tennessee, De- } \\
\text { partment of Pediatrics }\end{array}$ & $\begin{array}{l}\text { http://www.utmem.edu/pediatrics/residency/ } \\
\text { Library/perinatal_infx2006.ppt }\end{array}$ & $\begin{array}{l}\text { PowerPoint presentation about the diag- } \\
\text { nosis and therapy of perinatal infections }\end{array}$ \\
\hline $\begin{array}{l}\text { Geneva Foundation for Medi- } \\
\text { cal Education and Research }\end{array}$ & $\begin{array}{l}\text { http://www.gfmer.ch/Guidelines/ } \\
\text { Obstetrics_gynecology_guidelines.php }\end{array}$ & $\begin{array}{l}\text { Links to prevalence symptomatology, im- } \\
\text { ages, and treatment guidelines about } \\
\text { ob/gyn infections }\end{array}$ \\
\hline $\begin{array}{l}\text { The Royal Women's Hospital } \\
\text { (RWH) }\end{array}$ & $\begin{array}{l}\text { http://rwh.org.au/nets/handbook/index.cfm?doc_id } \\
=4972 \text { \#rub_intro }\end{array}$ & Congenital infections information \\
\hline $\begin{array}{l}\text { National Women's Health at } \\
\text { Auckland City Hospital }\end{array}$ & $\begin{array}{l}\text { http://www.adhb.govt.nz/newborn/ } \\
\text { Guidelines/Infection/CongenitalInfection.htm }\end{array}$ & $\begin{array}{l}\text { Guidelines for investigation of suspected } \\
\text { cases }\end{array}$ \\
\hline The Hospital for Sick Children & $\begin{array}{l}\text { http://www.motherisk.org/prof/ } \\
\text { infectiousDiseases.jsp }\end{array}$ & $\begin{array}{l}\text { Issues about infectious diseases during } \\
\text { pregnancy }\end{array}$ \\
\hline Karolinska Institute & $\begin{array}{l}\text { http://www.mic.ki.se/MEDIMAGES.html\# } \\
\text { GynecologyObstetrics }\end{array}$ & $\begin{array}{l}\text { Useful links to images, illustrations, and } \\
\text { case presentations }\end{array}$ \\
\hline Guttmacher Institute Practical & http://www.guttmacher.org & $\begin{array}{l}\text { Issues and reports from all around the } \\
\text { world }\end{array}$ \\
\hline $\begin{array}{l}\text { National institute of Allergy } \\
\text { and Infectious Diseases }\end{array}$ & http://www3.niaid.nih.gov/research/topics & Overviews about the ob/gyn infections \\
\hline Clinical Microbiology Reviews & http://cmr.asm.org & Full-text articles \\
\hline
\end{tabular}


TABle 1: Continued.

\begin{tabular}{|c|c|c|}
\hline Website title & Website address & Comments \\
\hline Chlamydiae.com & http://www.chlamydiae.com & A site dedicated to chlamydia \\
\hline Candida corporation & http://www.candida.com & A site dedicated to candida \\
\hline Mycoplasma.com & http://www.mycoplasma.com & Links to sites dedicated to mycoplasma \\
\hline Ureaplasma.com & http://www.ureaplasma.com & Links to sites dedicated to mycoplasma \\
\hline $\begin{array}{l}\text { International Herpes Virus Fo- } \\
\text { rum }\end{array}$ & http://www.ihmf.org & $\begin{array}{l}\text { Slide lectures, diagnostic atlas, and man- } \\
\text { agement guidance for herpes infection }\end{array}$ \\
\hline Virology. net & http://www.virology.net & $\begin{array}{l}\text { Information and links about viruses in- } \\
\text { cluding those causing ob/gyn infections }\end{array}$ \\
\hline Wong's Virology & http://virology-online.com/presentations/index.htm & $\begin{array}{l}\text { PowerPoint presentations about congen- } \\
\text { ital viral infections, Herpes viruses, and } \\
\text { more ob/gyn related viruses }\end{array}$ \\
\hline $\begin{array}{l}\text { American Pregnancy Associ- } \\
\text { ation }\end{array}$ & $\begin{array}{l}\text { http://www.americanpregnancy.org/ } \\
\text { pregnancycomplications/index.htm }\end{array}$ & $\begin{array}{l}\text { General information about infections } \\
\text { during pregnancy }\end{array}$ \\
\hline $\begin{array}{l}\text { National Network of STD/HIV } \\
\text { Prevention Training Centers }\end{array}$ & http://depts.washington.edu/nnptc/index.html & STD clinical training \\
\hline $\begin{array}{l}\text { The National Women's Health } \\
\text { Information Center }\end{array}$ & http://www.4woman.gov & $\begin{array}{l}\text { Immunization, symptoms, and treatment } \\
\text { guidance }\end{array}$ \\
\hline Family Health Information & $\begin{array}{l}\text { http://www.fhi.org/training/en/modules/STD/tools. } \\
\text { htm }\end{array}$ & $\begin{array}{l}\text { PowerPoint presentation about STDs with } \\
\text { extra slide corresponding narrative }\end{array}$ \\
\hline $\begin{array}{l}\text { National network for immu- } \\
\text { nization information }\end{array}$ & http://www.immunizationinfo.org & $\begin{array}{l}\text { Guidance for immunization for pregnant } \\
\text { women, prevention of HPV, etc. }\end{array}$ \\
\hline $\begin{array}{l}\text { British Association for Sexual } \\
\text { Health and HIV }\end{array}$ & http://www.bashh.orgn & Site dedicated to sexual health and HIV \\
\hline Globalrph.com & http://www.globalrph.com & Clinician guide to drug therapy \\
\hline $\begin{array}{l}\text { Organization of Teratology In- } \\
\text { formation }\end{array}$ & http://otispregnancy.org/otis_fact_sheets.asp & Specialists fact sheets \\
\hline Perinatology.com & $\begin{array}{l}\text { http://www.perinatology.com/exposures/ } \\
\text { infectionlist.htm }\end{array}$ & $\begin{array}{l}\text { Information about infections, symptoms, } \\
\text { treatment options, etc., during preg- } \\
\text { nancy; useful links }\end{array}$ \\
\hline Thefetus.net & http://www.thefetus.net/listing.php?id=1718 & $\begin{array}{l}\text { Information about infections, symptoms, } \\
\text { ultrasound images, treatment options, } \\
\text { etc., during pregnancy }\end{array}$ \\
\hline $\begin{array}{l}\text { International Birth Defects In- } \\
\text { formation System }\end{array}$ & http://www.ibis-birthdefects.org/start/infect.htm & $\begin{array}{l}\text { Useful links to images, diagnostic tests } \\
\text { and treatment options about congenital } \\
\text { infections }\end{array}$ \\
\hline Wikipedia.org & http://en.wikipedia.org/wiki/Main_Page & Free online encyclopedia \\
\hline General Practice Notebook & http://www.gpnotebook.co.uk/homepage.cfm & $\begin{array}{l}\text { Interactive information about congenital } \\
\text { and ob/gyn infections }\end{array}$ \\
\hline Netterimages.com & http://www.netterimages.com/image & Netter medical illustration site \\
\hline AccessMyLibrary & http://www.accessmylibrary.com & $\begin{array}{l}\text { Online news, research, and information, } \\
\text { free registration }\end{array}$ \\
\hline
\end{tabular}

\section{REFERENCES}

[1] M. E. Falagas and E. A. Karveli, "World Wide Web resources on antimicrobial resistance," Clinical Infectious Diseases, vol. 43, no. 5, pp. 630-633, 2006.

[2] G. Pappas and M. E. Falagas, "Free internal medicine case-based education through the World Wide Web: how, where, and with what?" Mayo Clinic Proceedings, vol. 82, no. 2, pp. 203-207, 2007.

[3] G. Pappas, P. Papadimitriou, and M. E. Falagas, "World Wide Web hepatitis B virus resources," Journal of Clinical Virology, vol. 38, no. 2, pp. 161-164, 2007. 


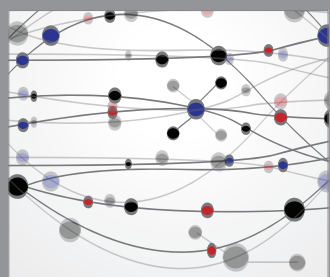

The Scientific World Journal
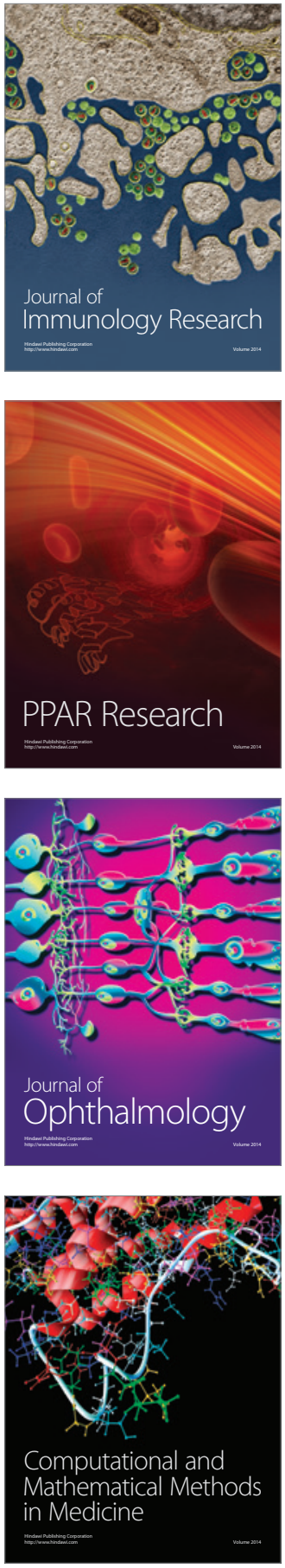

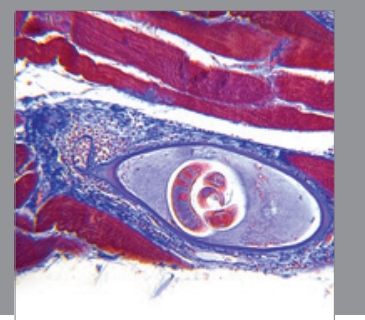

Gastroenterology

Research and Practice
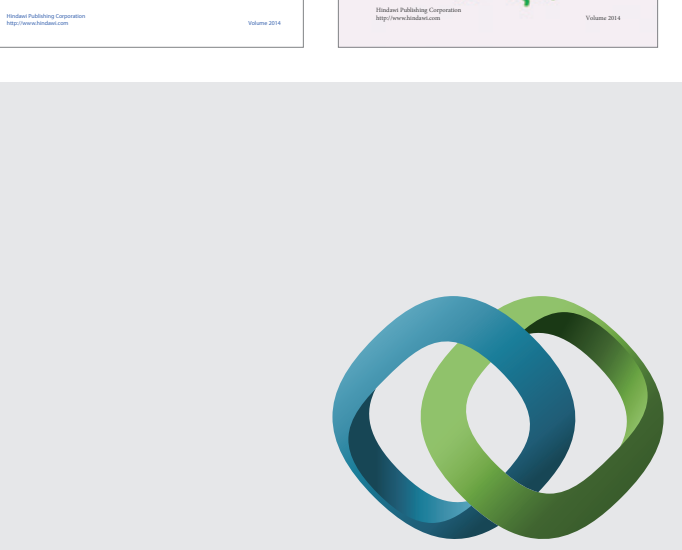

\section{Hindawi}

Submit your manuscripts at

http://www.hindawi.com
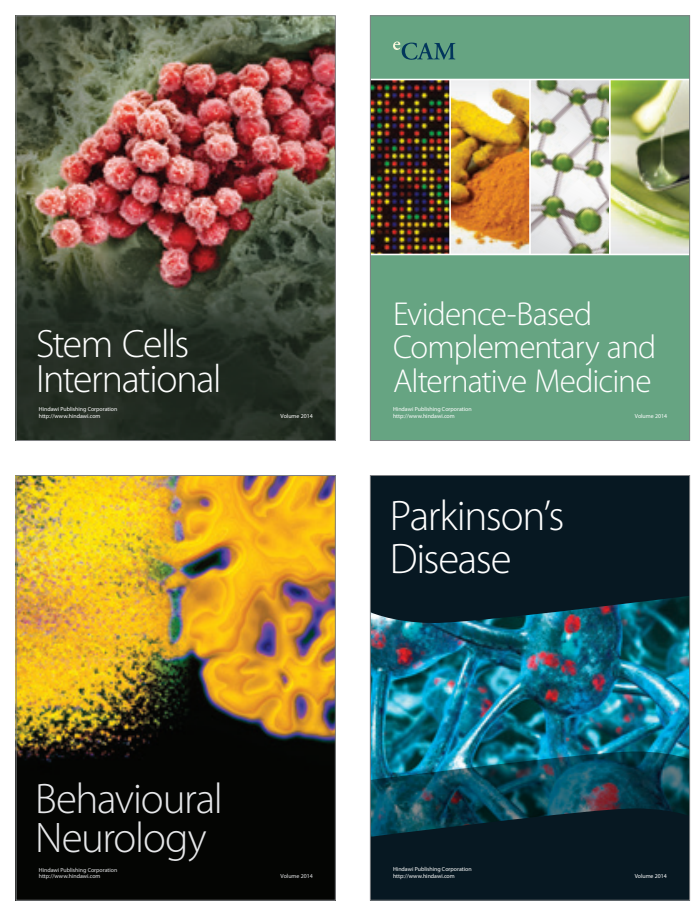

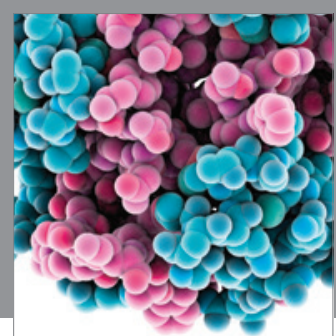

Journal of
Diabetes Research

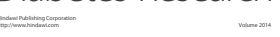

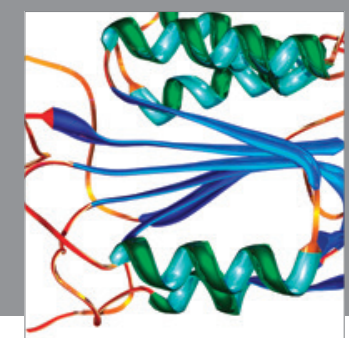

Disease Markers
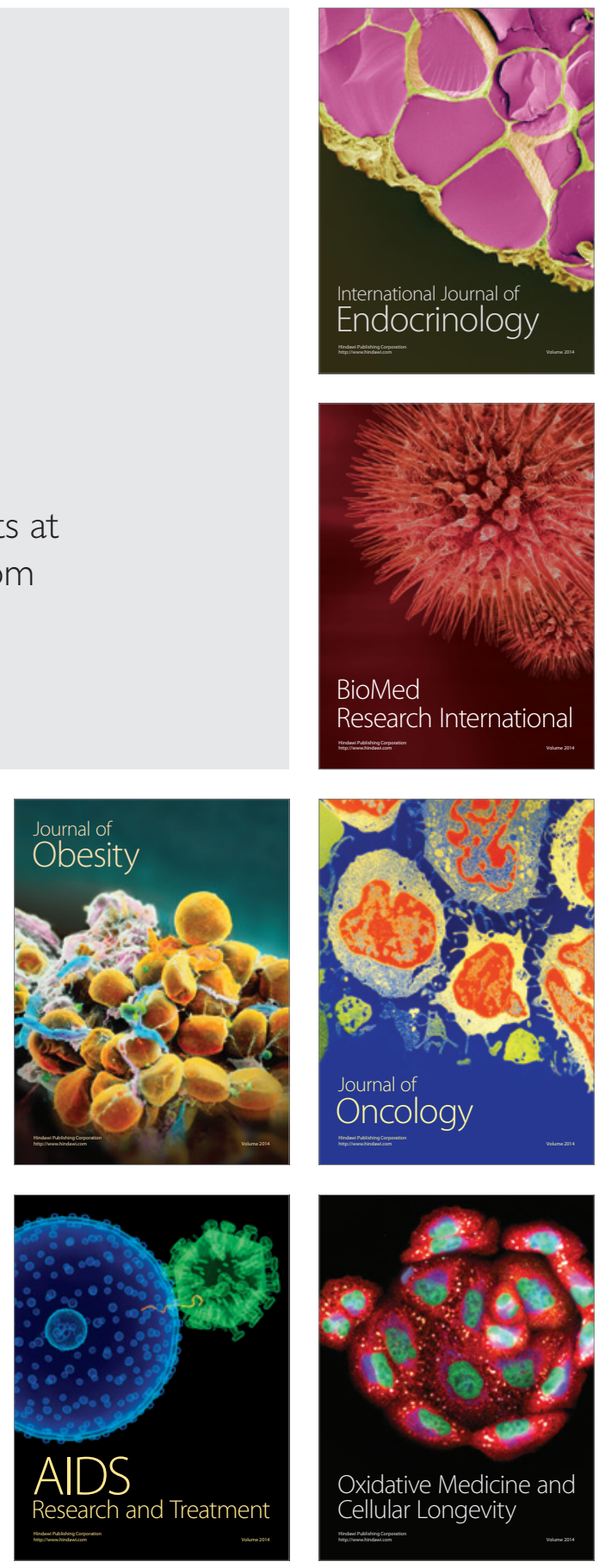